\title{
Hesperioidea e Papilionoidea (Lepidoptera) coligidos em expedição aos Rios Nhamundá e Abacaxis, Amazonas, Brasil: novos subsídios para o conhecimento da biodiversidade da Amazônia Brasileira
}

\author{
Mirna M. Casagrande ${ }^{1,3,4}$, Olaf H. H. Mielke ${ }^{1,3}$, Eduardo Carneiro ${ }^{1,3}$, José A. Rafael ${ }^{2,3}$ \& Roger W. Hutchings ${ }^{2}$ \\ ${ }^{1}$ Laboratório de Estudos de Lepidoptera Neotropical, Departamento de Zoologia, Universidade Federal do Paraná, Caixa Postal 19020, \\ 81531-980 Curitiba-PR, Brasil. \\ ${ }^{2}$ Instituto Nacional de Pesquisas da Amazônia, Av. André Araújo, 2936, 69060-001 Manaus-AM, Brasil. \\ ${ }^{3}$ Pesquisador/Bolsista CNPq. \\ ${ }^{4}$ Autor para correspondência, mibras@ufpr.br
}

\begin{abstract}
Hesperioidea and Papilionoidea (Lepidoptera) collected in an expedition to the Nhamundá and Abacaxis rivers, Amazonas, Brazil: new insights for understanding the biodiversity of the Brazilian Amazon. Aiming at improving the knowledge of the diurnal lepidopterofauna of the Amazon rainforest, this study lists 180 taxa collected at five different points along two tributaries of the Amazon River, including the Guyana and Rondonia areas of endemism. The different localities were compared using Non-Metric Multidimensional Scaling analysis (NMDS).
\end{abstract}

KEYWORDS. Brazilian Amazon; butterflies; distribution; endemism areas.

RESUMO. Hesperioidea e Papilionoidea (Lepidoptera) coligidos em expedição aos Rios Nhamundá e Abacaxis, Amazonas, Brasil: novos subsídios para o conhecimento da biodiversidade da Amazônia Brasileira. Objetivando um aprimoramento do conhecimento da lepidopterofauna diurna da Amazônia brasileira, este estudo lista 180 taxa coligidos em cinco pontos distintos de dois afluentes do Rio Amazonas, envolvendo as áreas de endemismo Guiana e Rondônia. As coletas foram passivas e ativas e as diferentes localidades comparadas através de análise de Escalonamento Multidimensional Não-Métrico (NMDS).

PALAVRAS-CHAVE. Amazônia brasileira; áreas de endemismo; borboletas; distribuição.

Até o presente, inventários faunísticos que objetivam listar as espécies de borboletas da Região Amazônica são escassos e, portanto, altamente prioritários para o Brasil (Santos et al. 2008). Apesar de ter sido, muitas vezes, explorada e com seus resultados publicados desde antigas comunicações científicas no final do século XIX (p. ex. Butler 1877; Sharpe 1890 , etc.), os inventários realizados na Amazônia brasileira costumeiramente carecem de uma descrição precisa das localidades de coleta (p. ex. margem do rio ou distância percorrida), o que restringe significativamente o uso de seus dados. Por vezes, tais estudos foram conduzidos através de longas viagens pelos grandes rios onde se amostravam diversas localidades, e em cujas referências consta apenas o nome do rio percorrido (p.ex. Butler 1877; Sharpe 1890; Ebert 1965). Wallace (1852), que já enfatizava a importância de indicar com exatidão a localidade de coleta na Amazônia, foi o primeiro a identificar diferenças significativas na sua fauna e relacioná-las às barreiras geográficas produzidas pelos grandes rios. Posteriormente, estas áreas passaram a ser denominadas áreas de endemismo (Cracraft 1985; Silva et al. 2002; Silva \& Garda 2011), para então serem utilizadas como base para formação de hipóteses de biogeografia histórica. Recentemente, Haseyama \& Carvalho (2011) através de uma abordagem evolutiva sobre os padrões de distribuição da biodiversidade Amazônica enfatizam a necessidade de obtenção de dados de localização geográfica das espécies que, associados a outros fatores, alimentam a dinâmica da biogeografia.

Sobre a fauna de borboletas, relacionada em forma de listas ou inventários, a área de endemismo Guiana talvez seja a que agrega mais informações para o Brasil: parte do trabalho de Butler (1877) com a lista de lepidópteros coletados por W. H. Trail durante dois anos em diversas localidades ao longo do rio Amazonas e seus afluentes, incluindo o Rio Nhamundá (como Jamunda), Amazonas; Rosa (1926) que relaciona 35 exemplares de lepidópteros doados ao Museu Nacional do Rio de Janeiro pela "Colônia Cleveland" no Oiapoque, Amapá; D’Almeida (1937) com resultados após excursão aos Rios Cuminá e Trombetas, Pará; Ebert (1965) relaciona as espécies coletadas próximas ao Rio Amapari, Amapá; Mielke (1973) com uma relação de Hesperioidea do Amapá e Pará, e Mielke \& Casagrande (1992) com a relação de espécies da Ilha de Maracá, Roraima, porém com a somatória de todos os registros conclui-se que estes dados são escassos perante o potencial estimado. Brown \& Hutchings (1997) registraram um total de 455 espécies ao longo de quinze anos de estudos em fragmentos de floresta amazônica ao Norte de Manaus, com um significativo esforço de 1239 horas, no entanto, como a proposta dos autores não era exatamente um inventário, não há registros específi- 
cos; os exemplares foram quantificados para indicativos de famílias e/ou subfamílias.

O conhecimento da lepidopterofauna, para a área de endemismo Rondônia, que inclui o Rio Abacaxis, conta com apenas dois inventários, ou sejam, os registros da segunda parte do trabalho de Butler (1877), inclusive no próprio Rio Abacaxis, e mais recentemente Emmel \& Austin (1990) em uma área intensamente amostrada no estado de Rondônia.

\section{MATERIAL E MÉTODOS}

Como parte do Projeto "Amazonas: diversidade de insetos ao longo de suas fronteiras", entre os dias 16/V a 4/VI, 2008, foram realizadas coletas ao Norte e Sul do Rio Amazonas, em parte de dois de seus tributários, Rios Nhamundá e Abacaxis, respectivamente. Foram coletados 527 exemplares de 180 taxa de borboletas. Cinco foram os pontos de coletas (Fig. 1), detalhados a seguir: Ponto (1) 16-19/V - Igarapé Areia, 17m, Rio Nhamundá - margem direita, $124 \mathrm{~km}$ NO de Nhamundá, Amazonas. 01³5'11's, 57³7'34”W; Ponto (2) 20-22/V Lago Cuipiranga, 9m, Rio Nhamundá - margem esquerda, 53 km NO de Nhamundá, Amazonas. 01 53 '58'S, 5702'59'W; Ponto (3) 26-28/V - Trilha da Pirarara, 26m, Rio Abacaxis margem direita, $137 \mathrm{~km}$ SE de Borba, Amazonas. 05¹5'17'S, 58 41'58'W; Ponto (4) 29-31/V - Campina Pacamari, 20m, Rio Abacaxis - margem direita, $150 \mathrm{~km}$ SO de Maués, Amazonas. 04³5'49”'S, 58¹3'14”W; Ponto (5) 2-4/VI - Comunidade Paxiúba, Rio Abacaxis - margem esquerda, $11 \mathrm{~km} \mathrm{E}$ de Borba, Amazonas. 04²8'48'S, 58³4'24'W

Na Tabela I, além dos dados destas coletas, também foram incluídos dados pregressos de espécies citadas em inventários ou listas. A nomenclatura foi atualizada e após o nome da espécie segue a inicial do autor que fez a citação.
Estas iniciais seguem aos números dos pontos acima citados, quando os taxa são coincidentes com os resultados deste estudo: B (Butler 1877), R (Rosa 1926), A (D’Almeida 1937), E (Ebert 1965), M (Mielke 1973), EA (Emmel \& Austin 1990) e MC (Mielke \& Casagrande 1992).

Todas as coletas foram realizadas em floresta de terra firme, exceto pelo ponto 4 - Campina Pacamari, que é uma área mais aberta que as demais e com vegetação mais baixa. Os demais pontos foram explorados através de trilhas pré-existentes em locais abandonados, áreas pouco antropizadas ou, trilhas abertas na ocasião, dentro da Floresta Ombrófila Densa.

Para as coletas foram utilizadas três metodologias; 1) busca ativa, com o uso de rede entomológica, percorrendo as trilhas e adjacências como beira dos rios e entorno de moradias quando em áreas com habitações; 2) através de armadilhas para Lepidoptera (padrão DeVries 1987), com iscas de peixe em decomposição e bananas fermentadas em caldo de cana, penduradas desde $10 \mathrm{~cm}$ do solo até no máximo 3 metros de altura e, 3) através da técnica de Ahrenholz, que usa pequenos pedaços de papel absorvente branco, umedecidos com saliva e depositados sobre a face adaxial de folhas da vegetação rasteira ou no máximo a meio metro do chão (Lamas et al. 1993). Todos os procedimentos foram realizados durante o dia, entre às $08 \mathrm{~h} 00 \mathrm{~min}-18 \mathrm{~h} 00 \mathrm{~min}$, variando para pouco mais ou pouco menos dependendo da luz do sol e presença ou ausência de chuvas. As armadilhas com isca permaneciam durante a noite, objetivando a coleta de espécies de hábitos crepusculares e foram vistoriadas pelas manhãs e finais de tarde.

A composição de espécies resultante de cada localidade de coleta foi comparada através de uma análise de ordenamento "Non-Metric Multimensional Scaling" (NMDS) junto a três outros inventários realizados nas mesmas regiões de endemismo: Rondônia (Emmel \& Austin 1990) e Guiana

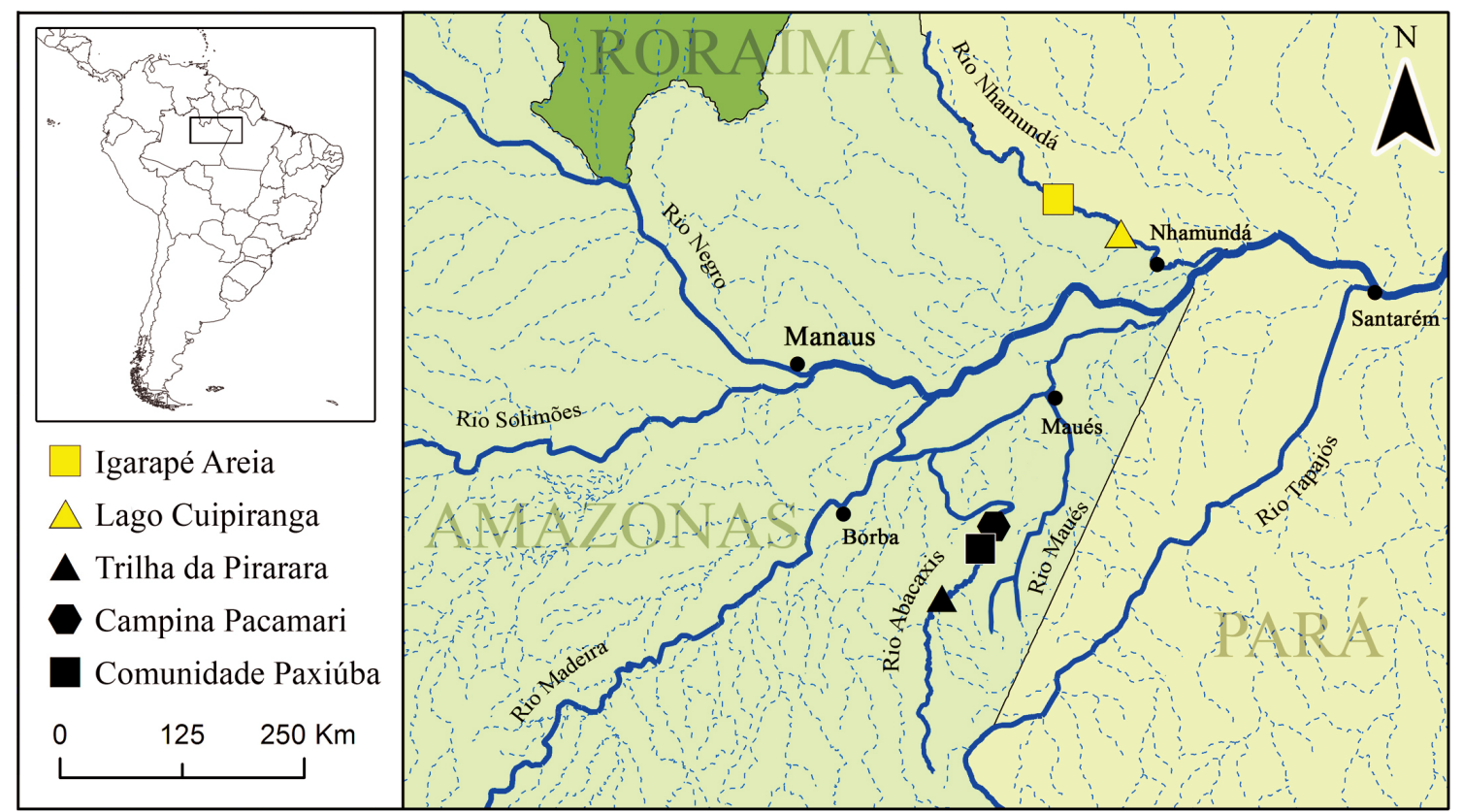

Figura 1. Mapa evidenciando as cinco localidades amostradas. 
Tabela I. Relação das espécies coletadas em cada ponto: (1) Igarapé Areia, (2) Lago Cuipiranga, (3) Trilha Pirarara, (4) Campina Pacamari, (5) Comunidade Paxiúba.Pontos 1 e 2, Rio Nhamundá (Área de Endemismo Guiana). Pontos 3-5, Rio Abacaxis (Área de Endemismo Rondônia). Registros em estudos pregressos são indicados pelas letras: B (Butler), R (Rosa), A (D’Almeida), E (Ebert), M (Mielke), EA (Emmel \& Austin) e MC (Mielke \& Casagrande).

\begin{tabular}{c} 
Espécies \\
\cline { 2 - 2 }
\end{tabular}

\section{Papilionoidea \\ Papilionidae \\ Papilioninae}

Leptocircini

Eurytides callias (Rothschild \& Jordan, 1906)

Protesilaus glaucolaus leucas (Rothschild \& Jordan, 1906)

Protesilaus protesilaus protesilaus (Linnaeus, 1758)

Protesilaus telesilaus telesilaus (C. Felder \& R.

Felder, 1864)

Mimoides ariarathes gayi (Lucas, 1852)

Troidini

$$
\text { Troidina }
$$

Parides neophilus ecbolius (Rothschild \& Jordan, 1906)

Parides vertumnus vertumnus (Cramer, 1779)

Pieridae

Coliadinae

Anteos menippe (Hübner, [1818])

Aphrissa statira statira (Cramer, 1777)

Aphrissa fluminensis (d'Almeida, 1921)

Phoebis argante argante (Fabricius, 1775)

Rhabdodryas trite trite (Linnaeus, 1758)

Pierinae

Anthocharidini

Hesperocharis nera amazonica Fruhstorfer, 1907 Pierin

Glutophrissa drusilla drusilla (Cramer, 1777)

Perrhybris pamela amazonica Fruhstorfer, 1907

Nymphalidae

Ithomiinae

Melinaeini

Melinaea ludovica ludovica (Cramer, 1780)

Melinaea mneme mneme (Linnaeus, 1763) Mechanitini

Methona confusa confusa Butler, 1873

Methona megisto C. Felder \& R. Felder, 1860

Scada reckia philemon C. Felder \& R. Felder, 1865

Mechanitis polymnia polymnia (Linnaeus, 1758) Napeogenini

Hypothyris euclea barii (Bates, 1862)

Hypothyris xanthostola xanthostola (Bates, 1862) Oleriini

Oleria similigena ssp. Dircennini

Callithomia lenea lenea (Cramer, 1779)

Morphinae

Morphini

Antirrheina

Antirrhea philoctetes murena Staudinger, [1886] Morphina

Morpho achilles achilles (Linnaeus, 1758)

Morpho deidamia deidamia (Hübner, [1819])
Opsip

$\begin{array}{ccc}\text { A } & 3,4 & \\ \text { B, A, MC } & 3,4 & \\ \text { A } & 3,4 & \\ & 3,4 & \text { P } \\ & & \end{array}$

$\begin{array}{cc}\text { 2, A } \\ \text { 1, 2, E } & \text { EA } \\ & \\ 2, \mathrm{~A} & \mathrm{EA} \\ \mathrm{A}, \mathrm{MC} & 3,4, \mathrm{~B}, \mathrm{EA} \\ 2 & 3,4 \\ \mathrm{R}, \mathrm{A}, \mathrm{E}, \mathrm{MC} & 3,4, \mathrm{EA} \\ \mathrm{A} & 3,4, \mathrm{EA}\end{array}$

$$
\text { Haeterini }
$$

Cithaerias andromeda bandusia Staudinger, [1887]

Haetera piera piera (Linnaeus, 1758)

Pierella hortona hortona (Hewitson, 1854)

Pierella hyalinus hyalinus (Gmelin, [1790])

Pierella lamia lamia (Sulzer, 1776)

Pierella lena lena (Linnaeus, 1767)

\section{Satyrini}

\section{Euptychiina}

Chloreuptychia chlorimene (Hübner, [1819])

Chloreuptychia herseis (Godart, [1824])

Cissia lesbia (Staudinger, [1886])

Cissia penelope (Fabricius, 1775)

Cissia terrestris (Butler, 1867)

Erichthodes antonina (C. Felder \& R. Felder, 1867)

Euptychia mollina (Hübner, [1813])

Hermeuptychia hermes (Fabricius, 1775)

Magneuptychia gera gera (Hewitson, 1850)

Magneuptychia gera nortia (Hewitson, 1862)

Magneuptychia harpyia batesii (Butler, 1867)

$\begin{array}{cc}\mathrm{A}, \mathrm{E}, \mathrm{MC} & 4, \mathrm{EA} \\ 1 & 5\end{array}$

Magneuptychia lea philippa (Butler, 1867)

Magneuptychia libye (Linnaeus, 1767)

Magneuptychia modesta (Butler, 1867)

Pseudodebis valentina (Cramer, 1779)

Splendeuptychia furina (Hewitson, 1862)

$1, \mathrm{E} \quad 3,5$

$1, \mathrm{E}$

2, B, A, MC

$$
2, \mathrm{E}
$$

1

$1, \mathrm{E}, \mathrm{MC}$

$1,2, \mathrm{E}, \mathrm{MC}$

1,2

1,2

$1, \mathrm{MC}$

EA

Taygetis laches laches (Fabricius, 1793

Taygetis thamyra (Cramer, 1779)

Taygetis rufomarginata Staudinger, 1888

Taygetis virgilia (Cramer, 1776)

$$
\text { Incertae sedis }
$$

Amphidecta calliomma (C. Felder \& R. Felder, 1862)

\section{Charaxinae}

Preponini

Archaeoprepona demophon demophon (Linnaeus, 1758)

Prepona laertes octavia Fruhstorfer, 1905

$$
\text { Biblidinae }
$$

Cyrestini
Marpesia chiron marius (Cramer, 1779)

Biblidini

$$
\text { Epicaliina }
$$

5, EA

E

Eunica amycla (Godart, [1824])

Eunica malvina malvina Bates 1864

Eunica sophonisba agele Seitz, 1915
Pierella astyoche astyoche (Erichson, [1849])

\begin{tabular}{cc}
\multicolumn{2}{c}{ Área de Endemismo } \\
\hline Guiana & Rondônia \\
\hline 1, R, MC & 5, EA \\
5
\end{tabular}

A, MC

3,5, EA

$1,2, \mathrm{E}$

$2 \quad$ EA

2 EA

$\begin{array}{cc}1, \mathrm{R}, \mathrm{A}, \mathrm{E} & 5 \\ 1, \mathrm{~A} & 5, \mathrm{EA} \\ & 3, \mathrm{EA} \\ \text { 1, 2, A, E, MC } & 3, \mathrm{EA} \\ \mathrm{A}, \mathrm{E} & 5, \mathrm{EA}\end{array}$

1, 2, B, A, E, MC 3, 4, 5, EA

$2,3 \quad 5$

1, B, A, E, MC 4, 5, EA

1

1, A, E, MC EA

1, 2, E, MC EA

2

B, R, A 3, 5

1, A, E, MC 5, B, EA

1,2

3,5, EA

$15, \mathrm{EA}$

5, EA

1, A, E, MC EA

2

15

2
$\mathrm{~A}, \mathrm{MC}$

E, MC 3

$\mathrm{B}, \mathrm{A}, \mathrm{MC} \quad 5, \mathrm{EA}$

2, B, A, MC EA

5

$\mathrm{R}, \mathrm{A}, \mathrm{E}, \mathrm{MC} \quad 4, \mathrm{EA}$

2

B, A, MC 3, EA

Catonephele acontius acontius (Linnaeus, 1771) B, R, A,MC 5, EA

4

B

3, EA

5

Continua 
Tabela I. Continuação.

Espécies
Epiphilina
Pyrrhogyra amphiro amphiro Bates, 1865
Pyrrhogyra crameri nautaca Frustorfer, 1908
Temenis pulchra amazonica Fruhstorfer, 1907
Callicorina
Callicore astarte astartoides (Dillon, 1948)
Nymphalinae
Coeini
Baeotus aeilus (Stoll, 1780)
Colobura dirce dirce (Linnaeus, 1758)
Historis acheronta acheronta (Fabricius, 1775)
Tigridia acesta tapajona (Butler, 1873)
Kallimini
Junonia genoveva genoveva (Cramer, 1780)
Limenitidinae
Limenitidini
Adelpha cocala cocala (Cramer, 1779)
Adelpha mesentina (Cramer, 1777)
Adelpha plesaure phliassa (Godart, [1824]
Heliconiinae
Heliconiini
Dryas iulia atcionea (Crat

Dryas iulia alcionea (Cramer, 1779)

Neruda aeode aeode (Hübner, [1813])

Heliconius antiochus antiochus (Linnaeus, 1767)

Heliconius erato amazona Staudinger, 1897

Heliconius melpomene meriana J.R.G. Turner, 1

1967

Heliconius numata superioris Butler, 1875

Riodinidae

Euselasiinae

Euselasiin

Euselasia bilineata Lathy, 1926

Euselasia uria uria (Hewitson, [1853])

Euselasia sp. 1

Euselasia sp. 2

Riodininae

Mesosemiin

Mesosemiina

Eunogyra satyrus Westwood, 1851

Leucochimona hyphea hyphea (Cramer, 1776)

Mesophthalma idotea Westwood, 1851

Mesosemia cippus Hewitson, 1859

Mesosemia lacernata Stichel, 1909

Mesosemia melaene melaene Hewitson, 1859

Mesosemia nyctea nyctea (Hoffmannsegg, 1818)

Mesosemia thymetus thymetus (Cramer, 1777)

Semomesia croesus meana (Hewitson, 1858)

Pterophthalma tullius (Fabricius, 1787)

$$
\text { Eurybiini }
$$

Eurybia nicaeus nicaeus (Fabricius, 1775)

$$
\text { Riodinini }
$$

Detritivora cleonus (Stoll, 1781)

Detritivora zama (Bates, 1868)

Isapis agyrtus mithrophorus Seitz, 1913 Symmachiini

Mesene phareus (Cramer, 1777)

Symmachia pardalis Hewitson, 1867

\begin{tabular}{lr}
\multicolumn{2}{l}{ Área de Endemismo } \\
\hline Guiana $\quad$ Rondônia
\end{tabular}

EA, 3

EA, 5

3

2

$\begin{array}{ll}\text { 2, R, A, E, MC } & 5 \\ \text { A, MC } & \text { EA } \\ 1 & 3,5 \\ & 3,5 \\ \text { 1, MC } & \text { B }\end{array}$

$\begin{array}{ll}\mathrm{A}, \mathrm{E}, \mathrm{MC} & 3, \mathrm{EA} \\ 1, \mathrm{E} & \mathrm{EA} \\ 1, \mathrm{MC} & \end{array}$

$\begin{array}{ll}\text { 1, B, A, E, MC } & \text { EA } \\ \text { A } & 3, \text { EA } \\ \text { B, A, E, MC } & 3 \\ & 3,4,5\end{array}$

1

1
$2, \mathrm{~A}, \mathrm{E}$
1
1

$\begin{array}{ll}1, \mathrm{~A} & 5, \mathrm{EA} \\ 1, \mathrm{MC} & 5, \mathrm{EA} \\ 1, \mathrm{~A} & \mathrm{EA} \\ & 5, \mathrm{EA} \\ 1, \mathrm{~A}, \mathrm{E} & 5 \\ & \text { EA } \\ & 5 \\ & 4\end{array}$

\begin{tabular}{|c|c|}
\hline $1,2, \mathrm{MC}$ & 5 , EA \\
\hline $1,2, \mathrm{E}, \mathrm{A}$ & $3,4, \mathrm{EA}$ \\
\hline $2, \mathrm{~A}$ & EA \\
\hline 2 & \\
\hline $1, \mathrm{~A}, \mathrm{MC}$ & EA \\
\hline
\end{tabular}

1, A, E, MC

Continua

\begin{tabular}{|c|c|c|}
\hline \multirow{2}{*}{ Espécies } & \multicolumn{2}{|c|}{ Área de Endemismo } \\
\hline & Guiana & Rondônia \\
\hline \multicolumn{3}{|l|}{ Helicopini } \\
\hline Sarota gyas (Cramer, 1775) & & 4, EA \\
\hline \multicolumn{3}{|l|}{ Nymphidiini } \\
\hline \multicolumn{3}{|l|}{ Lemoniadina } \\
\hline Lemonias zygia zygia Hübner, [1807] & $2, \mathrm{~B}, \mathrm{~A}$ & EA \\
\hline Juditha azan azan (Westwood, 1875) & 1 & \\
\hline Synargis abaris (Cramer, 1776) & $1, \mathrm{~A}, \mathrm{E}$ & \\
\hline \multicolumn{3}{|l|}{ Nymphidiina } \\
\hline Adelotypa aristus cretata (Stichel, 1911) & & 5 \\
\hline Adelotypa huebneri huebneri (Butler, 1867) & $\mathrm{A}, \mathrm{E}$ & 5 \\
\hline Adelotypa leucophaea (Hübner, [1821]) & 1 & EA \\
\hline Adelotypa penthea penthea (Cramer, 1777) & $1, \mathrm{~A}, \mathrm{E}$ & 4 \\
\hline Nymphidium acherois acherois (Boisduval, 1836) & $\mathrm{A}, \mathrm{E}$ & 5 \\
\hline Nymphidium aurum Callaghan, 1985 & $2, \mathrm{MC}$ & \\
\hline $\begin{array}{l}\text { Nymphidium azanoides amazonensis Callaghan, } \\
1986\end{array}$ & $1, \mathrm{MC}$ & \\
\hline Nymphidium baeotia Hewitson, [1853] & $\mathrm{B}, \mathrm{A}, \mathrm{E}, \mathrm{MC}$ & $3,5, \mathrm{EA}$ \\
\hline Nymphidium caricae caricae (Linnaeus, 1758) & $1, \mathrm{~B}, \mathrm{~A}, \mathrm{E}, \mathrm{MC}$ & 3, EA \\
\hline Nymphidium chione H.W. Bates, 1867 & A & 5 , EA \\
\hline Nymphidium leucosia galactina Seitz, 1917 & & 5 \\
\hline Nymphidium menalcus (Stoll, 1782) & $1,2, \mathrm{~A}, \mathrm{E}$ & \\
\hline Setabis epitus epitus (Cramer, 1780) & & 5 \\
\hline \multicolumn{3}{|l|}{ Theopina } \\
\hline Theope leucanthe Bates, 1868 & 1 & \\
\hline \multicolumn{3}{|l|}{ Stalachtini } \\
\hline Stalachtis calliope calliope (Linnaeus, 1758) & $\mathrm{B}, \mathrm{A}, \mathrm{MC}$ & 3, EA \\
\hline Stalachtis euterpe euterpe (Linnaeus, 1758) & $1, \mathrm{~B}, \mathrm{~A}$ & B \\
\hline Stalachtis lineata (Guérin-Méneville, [1844]) & $2, \mathrm{~B}, \mathrm{~A}$ & 3 \\
\hline Stalachtis phaedusa phaedusa (Hübner, [1813]) & $\mathrm{A}, \mathrm{E}$ & 3,5 \\
\hline Stalachtis phaedusa zephyrits (Dalman, 1823) & 1 & \\
\hline Stalachtis phlegia phlegetontia (Perty, 1833) & $1, \mathrm{~B}, \mathrm{~A}$ & B \\
\hline \multicolumn{3}{|l|}{ Lycaenidae } \\
\hline \multicolumn{3}{|l|}{ Theclinae } \\
\hline \multicolumn{3}{|l|}{ Eumaeini } \\
\hline Arawacus aetolus (Sulzer, 1776) & $1, \mathrm{E}, \mathrm{MC}$ & 5 \\
\hline Arumecla aruma (Hewitson, 1877) & $1, \mathrm{E}$ & \\
\hline Badecla badaca (Hewitson, 1868) & 1 & \\
\hline Calycopsis cerata (Hewitson, 1877) & $1, \mathrm{E}$ & \\
\hline Calycopis malta (Schaus, 1902) & 1 & 3 \\
\hline Calycopis meleager (H. H. Druce, 1907) & & 5 \\
\hline Chalybs hassan (Stoll, 1790) & 1 & \\
\hline Panthiades bitias (Cramer, 1777) & $\mathrm{MC}$ & 3 \\
\hline Symbiopsis aprica (Möschler, 1883) & 1 & \\
\hline Theritas lisus (Stoll, 1790) & 1 & \\
\hline
\end{tabular}

Tabela I. Continuação.

Hesperioidea

\section{Hesperiidae \\ Pyrginae}

Eudamini

Augiades crinisus (Cramer, 1780)

Autochton neis (Geyer, 1832)

Autochton zarex (Hübner, 1818)

A, E, M, MC 3

1, 2, B, A, M, MC EA

$1, \mathrm{~A}, \mathrm{E}, \mathrm{M}, \mathrm{MC}$

1869)

Dyscophellus ramusis astrapaeus (Hewitson, 1876) 1, M

Entheus priassus priassus (Linnaeus, 1758) 2, M

Entheus sp. 1

Entheus sp. 2

3,5

Continua 
Tabela I. Continuação.

\begin{tabular}{|c|c|c|}
\hline \multirow{2}{*}{ Espécies } & \multicolumn{2}{|c|}{ Área de Endemismo } \\
\hline & Guiana & Rondônia \\
\hline Hyalothyrus leucomelas (Geyer, 1832) & $\mathrm{MC}$ & 5, EA \\
\hline Hyalothyrus infernalis infernalis (Möschler, 1877) & $1, \mathrm{~A}, \mathrm{M}$ & $4,5, \mathrm{EA}$ \\
\hline Oileides azines (Hewitson, 1867) & 1 & \\
\hline Proteides mercurius mercurius (Fabricius, 1787) & $\mathrm{A}, \mathrm{E}$ & 4 \\
\hline Urbanus esma Evans, 1952 & MC & 4 \\
\hline Urbanus velinus (Plötz, 1880) & M & 3 \\
\hline \multicolumn{3}{|l|}{ Pyrgini } \\
\hline Achlyodes busirus busirus (Cramer, 1779) & $1, \mathrm{M}$ & \\
\hline Pachyneuria duidae duidae (Bell, 1932) & & 3 \\
\hline Paramimus scurra scurra (Hübner, [1809]) & $1, \mathrm{M}$ & \\
\hline $\begin{array}{l}\text { Paramimus scurra herberti Godman \& Salvin, } \\
1896\end{array}$ & & $5, \mathrm{EA}$ \\
\hline Pythonides herennius herennius Geyer, [1832] & MC & 5 \\
\hline Telemiades amphion amphion (Geyer, 1832) & M & 3 \\
\hline Telemiades penidas (Hewitson, 1867) & 1 & \\
\hline Telemiades corbulo corbulo $($ Stoll, 1781$)$ & 2 & \\
\hline \multicolumn{3}{|l|}{ Hesperiinae } \\
\hline Anthoptus insignis (Plötz, 1882) & $1, \mathrm{M}, \mathrm{MC}$ & EA \\
\hline Artines focus Evans, 1955 & $2, \mathrm{MC}$ & \\
\hline Apaustus gracilis smarti Evans, 1955 & $1, \mathrm{M}$ & \\
\hline Carystoides sicania orbius (Godman, 1901) & 1 & EA \\
\hline Cobalus calvina (Hewitson, 1866) & M & $5, \mathrm{EA}$ \\
\hline Cymaenes alumna (Butler, 1877) & $1 \mathrm{E}, \mathrm{M}$ & $\mathrm{B}$ \\
\hline Damas clavus (Herrich-Schäffer, 1869) & $1, \mathrm{M}, \mathrm{MC}$ & EA \\
\hline Dubiella dubius (Stoll, 1781) & & $5, \mathrm{EA}$ \\
\hline Ebusus ebusus ebusus (Cramer, 1780) & $1, \mathrm{M}$ & \\
\hline $\begin{array}{l}\text { Eutychide subcordata subcordata (Herrich- } \\
\text { Schäffer, 1869) }\end{array}$ & $2, \mathrm{MC}$ & \\
\hline Morys compta compta (Butler, 1877) & $1, \mathrm{~B}, \mathrm{M}, \mathrm{MC}$ & EA \\
\hline Nyctelius nyctelius nyctelius (Latreille, [1824]) & $\mathrm{M}, \mathrm{MC}$ & 4 \\
\hline Panoquina lucas lucas (Fabricius, 1783) & $\mathrm{M}, \mathrm{MC}$ & 4, EA \\
\hline Parphorus decorus (Herrich-Schäffer, 1869) & $\mathrm{E}, \mathrm{MC}$ & 5 \\
\hline Phanes aletes (Geyer, 1832) & 2, M, MC & EA \\
\hline Saliana salius (Cramer, 1775) & $1, \mathrm{M}, \mathrm{MC}$ & \\
\hline Vettius lafrenaye pica (Herrich-Schäffer, 1869) & 2, $\mathrm{MC}$ & \\
\hline
\end{tabular}

(D’Almeida 1937; Mielke \& Casagrande 1992). Como uma análise de ordenamento, ela diminuiu a dimensionalidade dos atributos e conseqüentemente facilitou a comparação entre a composição faunística de cada ponto de coleta juntamente com os inventários pregressos. Como a análise utiliza índices de similaridade optamos pelo Índice de Dice (Wolda 1981), por ser o mais indicado quando se comparam diferentes tamanhos de amostras.

A classificação adotada segue Lamas (2004) e o material coletado está depositado nas coleções do Departamento de Zoologia, Universidade Federal do Paraná (Coleção Pe. Jesus Santiago Moure - Lepidoptera), Curitiba, Paraná e na Coleção de Invertebrados do Instituto Nacional de Pesquisas da Amazônia (INPA), Manaus, Amazonas.

\section{RESULTADOS E DISCUSSÃO}

Foram registrados um total de 180 taxa de borboletas ( 38 spp. Hesperioidea e $142 \mathrm{spp}$. Papilionoidea) durante toda a expedição, sendo destas 108 spp. no Rio Nhamundá (área de endemismo Guiana) e 101 spp. no Rio Abacaxis (área de endemismo Rondônia).

A diversidade de borboletas amazônicas ainda é esparsamente representada no Brasil e quando isso acontece os resultados são oriundos de estudos de curta duração, normalmente realizados em áreas ao longo dos grandes rios e/ou pequenos trechos de seus afluentes, através de excursões fluviais. Comparados a inventários de longa duração em áreas mais distantes e não somente dependentes de estreitas faixas nas margens de rios na região Amazônica (p. ex. Emmel \& Austin 1990; Robbins et al. 1996), o total de espécies encontradas no presente estudo é baixo, sugerindo que levantamentos ecológicos de curta duração são ineficazes quando objetivam investigar a magnitude e os padrões de distribuição da biodiversidade Amazônica. Uma grande parte dos registros aqui apresentados representa ocorrências inéditas em inventários para a Amazônia brasileira ou para áreas de endemismo amazônicas, fator que, concomitantemente, releva a necessidade de estudos de biodiversidade amazônicos e a carência de estudos na região.

Inventários não são as únicas fontes pelas quais podem ser obtidos registros da biodiversidade Amazônica. Diversas localidades foram consideravelmente amostradas, como São Paulo de Olivença, Tefé, Óbidos, Belém, etc., conforme material depositado em renomadas coleções nacionais e internacionais, e que pelo fato de estarem desta forma dispersa, dificultam o acesso e conseqüente reunião de informações. Uma urgente revisão na literatura e nas coleções científicas objetivando resgatar dados de estudos isolados como descrições de espécies, revisões de gêneros e similares é necessária para aprimorar as informações sobre o que se tem até o momento sobre a biodiversidade da Amazônia.

Agregamos a este levantamento os resultados obtidos por Butler (1877), Rosa (1926), D’Almeida (1937), Ebert (1965), Mielke (1973), Emmel \& Austin (1990) e Mielke \& Casagrande (1992), estudos estes realizados em locais relacionados com as áreas de endemismo Guiana e Rondônia e que se caracterizam como inventários ou listas. A nomenclatura foi atualizada com base em Lamas (2004) já que alguns nomes atualmente estão na sinonímia. Do total de 180 taxa, 58 são listados pela primeira vez em inventários na Amazônia brasileira, enquanto 39 taxa coletados no Rio Nhamundá são inéditos para a área de endemismo Guiana e 54 taxa no Rio Abacaxis, inéditos para a área de endemismo Rondônia. Desta forma, os resultados aqui apresentados representam uma ínfima parcela do conhecimento sobre a lepidopterofauna da Amazônia, tanto a já conhecida e totalmente dispersa, como aquela a ser levantada.

A análise de NMDS (Fig. 2) evidenciou uma maior influência do esforço amostral na ordenação das localidades, representada pelo primeiro eixo, onde as áreas mais amostradas, como Cacaulândia, Rondônia (Emmel \& Austin 1990); Maracá, Roraima (Mielke \& Casagrande 1992) e Rios Cuminá e Trombetas, Pará (D‘Almeida 1937) aparecem mais distanciadas dos pontos amostrados nos Rios Nhamundá e Abacaxis. No entanto, no segundo eixo, a composição diferencial das áreas de endemismo é melhor representada com os pontos 
amostrados para a área de endemismo Rondônia na parte superior e, Guiana na inferior. Desta forma, o uso de métodos de agrupamento espacial deve ser conduzido com cautela, em especial em localidades Amazônicas. Esforços mais intensos são necessários para amostrar sua diversidade, o que de fato corrobora com as observações ao longo de quinze anos de Brown \& Hutchings (1997) quando relatam que a cada nova visita às áreas amostradas, novas espécies eram incluídas, ao mesmo tempo em que, espécies já coletadas em visitas anteriores, nem sempre eram encontradas em visitas subseqüentes.

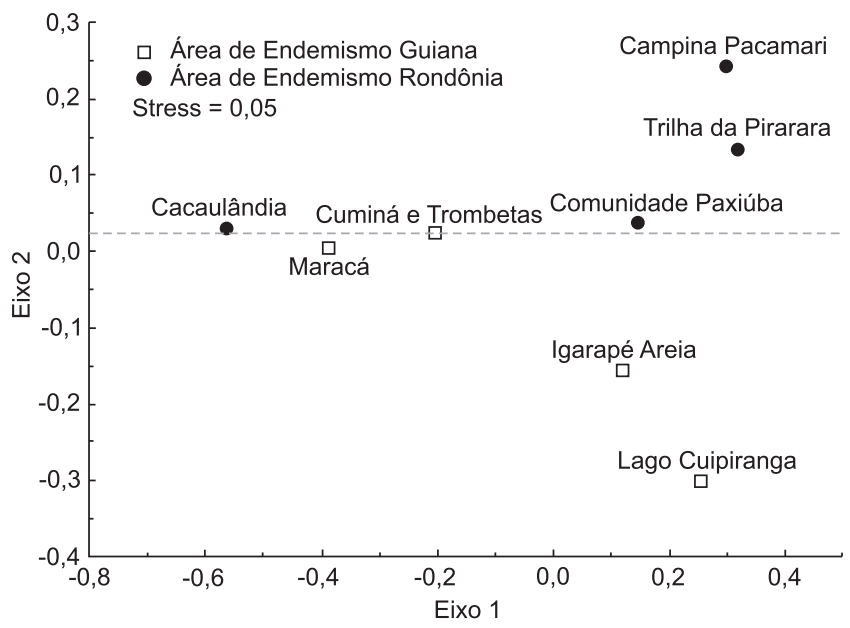

Figura 2. Análise de NMDS evidenciando a similaridade da lepidopterofauna encontrada nos pontos amostrais acrescidos a inventários pregressos nas regiões de endemismo Guiana e Rondônia.

\section{AGRADECIMENTOS}

Agradecemos à Fundação de Amparo à Pesquisa do Estado do Amazonas (FAPEAM) e ao Conselho Nacional de Desenvolvimento Científico e Tecnológico $(\mathrm{CNPq})$ pelo financiamento do projeto "Amazonas: diversidade de insetos ao longo de suas fronteiras" (Proc. 1437/2007) como parte do Programa de Apoio a Núcleos de Excelência (PRONEX); pelas bolsas de pesquisa (MMC, OHHM, JAR) e doutorado (EC); ao Dr. Marcelo Duarte, Museu de Zoologia, Universidade de São Paulo, SP, pelo auxílio na identificação de Lycaenidae e Dr. Maurício Moura, Departamento de Zoologia, Universidade Federal do Paraná, pelo auxílio na análise estatística. Estes resultados também fazem parte da RedLep "Rede Nacional de Pesquisa e Conservação de Lepidópteros" SISBIOTA-Brasil/CNPq (563332/2010-7). OHM é pesquisador Associado do McGuire Center for Lepidoptera and Biodiversity, Gainesville, FL, USA.

\section{REFERÊNCIAS}

Brown Jr., K. S. \& R. W. Hutchings. 1997. Disturbance, Fragmentation, and the Dynamics of Diversity in Amazonian Forest Butterflies, p. 91110. In: W. F. Laurance \& R. O. Bierregaard, Jr. (eds). Tropical Forest Remnants. Ecology, Management, and Conservation of Fragmented Communities. Chicago, The University of Chicago Press, xi+616 p.
Butler, A. G. 1877. On the Lepidoptera of the Amazons, collected by James W. H. Trail, Esq., during the years 1873 to 1875 . Transactions of the Entomological Society of London 1877: 105-156.

Cracraft, J. 1985. Historical biogeography and patterns of differentiation within the South American avifauna: areas of endemism. Ornithological Monographs 36: 49-84.

D’Almeida, R. F. 1937. Excursão scientifica aos rios Cuminá e Trombetas. Memórias do Instituto Oswaldo Cruz 32: 235-298.

DeVries, J. P. 1987. The butterflies of Costa Rica and their natural history. Papilionidae, Pieridae, Nymphalidae. Princeton, Princeton University Press, xxii+327 p.

Ebert, H. 1965. Uma coleção de borboletas (Lepid. Rhopal.) do Rio Amapari (território do Amapá) com anotações taxonômicas sobre Rhopalocera do Brasil. Papéis Avulsos do Departamento de Zoologia 18: 65-85.

Emmel, T. C. \& G. T. Austin. 1990. The tropical rain forest butterfly fauna of Rondonia, Brazil: species diversity and conservation. Tropical Lepidoptera 1: 1-12.

Haseyama, K. L. F. \& C. J. B. de Carvalho. 2011. Padrões de distribuição da biodiversidade Amazônica: um ponto de vista evolutivo. Revista da Biologia. Vol. Esp. Biogeografia: $35-40$.

Lamas, G. 2004. Checklist: Part 4a. Hesperioidea - Papilionoidea. In: J. B. Heppner (ed.) Atlas of Neotropical Lepidoptera. Volume 5A, Gainesville, Association of Tropical Lepidoptera, xxxv+439 p.

Lamas G., O. H. H. Mielke \& R. K. Robbins. 1993. The Ahrenholz technique for attracting tropical skippers (Hesperiidae). Journal of the Lepidopterists' Society 47: 80-82.

Mielke, O. H. H. 1973. Contribuição ao estudo dos Hesperiidae americanos. III Espécies coletadas em duas excursões ao Pará e Amapá, Brasil (Lepidoptera). Acta Biológica Paranaense, Curitiba, 2: 17-40.

Mielke, O. H. H. \& M. M. Casagrande. 1992. Lepidoptera: Papilionoidea e Hesperioidea coletados na Ilha de Maracá, Alto Alegre, Roraima, parte do projeto Maracá, com uma lista complementar de Hesperiidae de Roraima. Acta Amazonica 21: 175-210.

Robbins, R. K.; G. Lamas; O. H. H. Mielke; D. J. Harvey \& M. M. Casagrande. 1996. Taxonomic composition and ecological structure of the speciesrich butterfly community at Pakitza, Parque Nacional del Manu, Perú. p. 217-252. In: D. E. Wilson \& A. Sandoval (eds). Manu. The Biodiversity of Southeastern Peru. La Biodiversidad del Sureste del Perú. Smithsonian Institution, Washington, D.C., 679 p.

Rosa, M. 1926. Lepidópteros do Norte do Brasil. Boletim do Museu Nacional do Rio de Janeiro 2: 23-24.

Santos, E. C.; O. H. H. Mielke \& M. M. Casagrande. 2008. Inventários de borboletas no Brasil: estado da arte e modelo de áreas prioritárias para pesquisa com vistas à conservação. Natureza \& Conservação, Curitiba, 6: 68-90.

Sharpe. E. M. 1890. On a Collection of Lepidoptera made by Mr. Edmund Reynolds on the Rivers Tocantins and Araguaya and in the Province of Goyaz, Brasil. Proceedings of the Zoological Society of London 1890: 552-577.

Silva, J. M. C. \& A. A. Garda. 2011. Padrões e Processos Biogeográficos na Amazônia: 189-197. In: C. J. B. de Carvalho \& E. A. B. Almeida (Org.). Biogeografia da América do Sul: padrões e processos. São Paulo, Editora Roca, $306 \mathrm{p}$.

Silva, J. M. C. da; F. C. Novaes \& D. C. Oren. 2002. Differentiation of Xiphocolaptes (Dendrocolaptidae) across the river Xingu, Brazilian Amazonia: recognition of a new phylogenetic species and biogeographic implications. Bulletin of the British Ornithologists' Club 122: 185-194.

Wallace, A. R. 1852. On the monkeys of the Amazon. Proceedings of the Zoological Society of London 1852 (20): 107-110.

Wolda, H. 1981. Similarity indices, sample size and diversity. Oecologia 50: $269-302$.

Received 12/9/2011; accepted 11/2/2012

Editor: Marcelo Duarte 